\title{
Predicting sulfotyrosine sites using the random forest algorithm with significantly improved prediction accuracy Zheng Rong Yang
}

Address: School of Biosciences, University of Exeter, UK Exeter EX4 5DE, UK

Email: Zheng Rong Yang - z.r.yang@ex.ac.uk

Published: 29 October 2009

BMC Bioinformatics 2009, 10:361 doi:10.1186/|47|-2105-10-36|
Received: 4 August 2009

Accepted: 29 October 2009

This article is available from: http://www.biomedcentral.com//47/-2105/10/36I

(c) 2009 Yang; licensee BioMed Central Ltd.

This is an Open Access article distributed under the terms of the Creative Commons Attribution License (http://creativecommons.org/licenses/by/2.0), which permits unrestricted use, distribution, and reproduction in any medium, provided the original work is properly cited.

\begin{abstract}
Background: Tyrosine sulfation is one of the most important posttranslational modifications. Due to its relevance to various disease developments, tyrosine sulfation has become the target for drug design. In order to facilitate efficient drug design, accurate prediction of sulfotyrosine sites is desirable. A predictor published seven years ago has been very successful with claimed prediction accuracy of $98 \%$. However, it has a particularly low sensitivity when predicting sulfotyrosine sites in some newly sequenced proteins.

Results: A new approach has been developed for predicting sulfotyrosine sites using the random forest algorithm after a careful evaluation of seven machine learning algorithms. Peptides are formed by consecutive residues symmetrically flanking tyrosine sites. They are then encoded using an amino acid hydrophobicity scale. This new approach has increased the sensitivity by $22 \%$, the specificity by $3 \%$, and the total prediction accuracy by $10 \%$ compared with the previous predictor using the same blind data. Meanwhile, both negative and positive predictive powers have been increased by $9 \%$. In addition, the random forest model has an excellent feature for ranking the residues flanking tyrosine sites, hence providing more information for further investigating the tyrosine sulfation mechanism. A web tool has been implemented at http://ecsb.ex.ac.uk/ sulfotyrosine for public use.

Conclusion: The random forest algorithm is able to deliver a better model compared with the Hidden Markov Model, the support vector machine, artificial neural networks, and others for predicting sulfotyrosine sites. The success shows that the random forest algorithm together with an amino acid hydrophobicity scale encoding can be a good candidate for peptide classification.
\end{abstract}

\section{Background}

Tyrosine sulfation is a posttranslational modification (PTM), which introduces a sulfate group to a tyrosine residue in a protein [1-3]. During the modification process, sulfation is catalysed by tyrosylprotein sulfotransferase [4]. A targeted tyrosine for sulfation is normally required to be exposed on a protein surface [5]. Previous studies have indicated that Sulfation is an important anticipator for extracellular protein-protein interactions [6,7]. Studies have shown that sulfation is related to various diseases when a malfunction of a cellular activity occurs. For instance, sulfotyrosine can alter the affinity in some chemokine receptors leading to a downstream signalling cascade which affects the cells involved in acute and chronic events of cellular immunity [8]. Disease-related alterations at the non-reducing termini of chondroitin and dermatan sulfate have been found useful for monitoring proteoglycan metabolism [9]. In biochemistry, sulfation 
has been recognised as an important contributor to detoxication for endogenous compounds [10]. Sulfation activity has been investigated in various cancer studies such as breast cancer [11-13], lung cancer [14], prostate cancer $[15,16]$, and pancreatic cancer [17-19]. Because of the relevance to various disease, tyrosine sulfation has been the target for drug design for over a decade [20-25].

In silico prediction of posttranslational modification sites is a significant activity in bioinformatics. For instance, in ExPASy http://www.expasy.ch/tools various PTM site predictors have been implemented. Specifically, a predictor named as Sulfinator http://www.expasy.ch/tools/sulfina tor for sulfotyrosine site prediction has been successfully implemented using Hidden Markov Models (HMM) [26]. The predictor was able to obtain a sensitivity (the accuracy of predicting true sulfotyrosine sites) of $98 \%$ and total prediction accuracy of $98 \%$. When the predictor is used on newly sequenced proteins, it is found that the predictor has a particularly low sensitivity although the specificity (the accuracy of predicting unconfirmed sulfotyrosine sites) is high. In this study, a new approach is therefore developed aiming to improve the sensitivity while maintaining the specificity. There is another predictor developed only for tyrosine sulfation sites in animal viruses using Position-Specific-Scoring-Matrix (PSSM) [27]. This approach is very similar to the so-called $h$-function proposed by Poorman [28] 18 years ago. Because only positive peptides are used for scoring, such an approach suffers low specificity when used for making prediction on unseen data [29]. 69 Jackknife simulations were conducted for only positive data. Although it claimed prediction accuracy of $96.43 \%$, the model was actually trained with a carefully selected threshold. The claimed accuracy was observed after tuning the threshold, which is there-
Table I: The prediction result for the 15 blind test sequences using the Sulfinator.

\begin{tabular}{llllll}
\hline Accession & \multicolumn{2}{c}{ Actual sites } & \multicolumn{3}{c}{ Predicted sites } \\
\hline Q9PU41 & 112 & & 112 & \\
P6I073 & 21 & & 21 & \\
Q9NZ53 & 97 & 118 & 97 & & \\
A2ZBG5 & 110 & 112 & & & \\
A2YFB4 & 80 & $\mathbf{8 2}$ & 80 & & \\
Q7 M3V5 & 114 & & 114 & 102 & 131 \\
P84900 & $\mathbf{6 2}$ & & & & \\
Q0VTT9 & $\mathbf{6 2}$ & & & & \\
P0CIV8 & 16 & & 16 & & \\
P0CIV7 & 16 & & 16 & \\
Q800FI & 62 & & & \\
P68II6 & 5 & & 5 & \\
P68I24 & 4 & & 4 & \\
P68I21 & 3 & & 3 & \\
P68II9 & 6 & & 6 & \\
\hline
\end{tabular}

fore likely over-estimated. Meanwhile, there is no public available tool for the comparison.

In a review paper, some most common features describing the patterns of the residues flanking a tyrosine sulfation site were given [30]. The patterns are found from the residues which flank the experimentally verified tyrosine sulfation sites using a regular expression pattern match approach. This is commonly used in various posttranslational modification pattern analysis projects. The web tool called WebLogos (or sequence logos) is such an application [31]. The reviewer discussed some motif patterns summarised from an earlier study, for instance, Glu and Asp commonly occur between -2 and 2 of a tyrosine sulfation site. However, the regular expression approach suffers two theoretical limitations. First, such an approach

Table 2: The prediction performances of all machine learning models.

\begin{tabular}{|c|c|c|c|c|c|c|c|c|c|c|c|c|}
\hline & \multicolumn{4}{|c|}{10} & \multicolumn{4}{|c|}{20} & \multicolumn{4}{|c|}{30} \\
\hline & Spe & Sen & Tot & AUR & Spe & Sen & Tot & AUR & Spe & Sen & Tot & AUR \\
\hline LDA & 70 & 78 & 73 & 0.80 & 76 & 89 & 80 & 0.87 & 82 & 83 & 82 & 0.88 \\
\hline QDA & 85 & 50 & 73 & 0.82 & 88 & 44 & 73 & 0.80 & 91 & 72 & 84 & 0.84 \\
\hline CART & 91 & 72 & 84 & n.a. & 76 & 83 & 78 & n.a. & 88 & 83 & 86 & n.a. \\
\hline INN & 91 & 72 & 84 & n.a. & 88 & 72 & 82 & n.a. & 85 & 72 & 80 & n.a. \\
\hline $3 N N$ & 85 & 78 & 82 & n.a. & 94 & 72 & 86 & n.a. & 94 & 72 & 86 & n.a. \\
\hline $5 N N$ & 94 & 72 & 86 & n.a. & 97 & 72 & 88 & n.a. & 97 & 72 & 88 & n.a. \\
\hline $7 \mathrm{NN}$ & 88 & 67 & 78 & n.a. & 88 & 78 & 84 & n.a. & 94 & 78 & 88 & n.a. \\
\hline 9NN & 94 & 50 & 78 & n.a. & 88 & 78 & 86 & n.a. & 94 & 78 & 88 & n.a. \\
\hline $\mathrm{RF}$ & 97 & 83 & 92 & 0.93 & 97 & 83 & 92 & 0.95 & 97 & 83 & 92 & 0.94 \\
\hline ANN5 & 94 & 28 & 71 & 0.81 & 88 & 67 & 80 & 0.86 & 88 & 78 & 84 & 0.92 \\
\hline ANNIO & 100 & 33 & 76 & 0.82 & 94 & 78 & 88 & 0.94 & 91 & 72 & 84 & 0.92 \\
\hline ANNI5 & 91 & 56 & 78 & 0.86 & 97 & 67 & 86 & 0.89 & 94 & 78 & 88 & 0.93 \\
\hline ANN20 & 91 & 56 & 78 & 0.88 & 94 & 72 & 86 & 0.96 & 94 & 78 & 88 & 0.93 \\
\hline SVM & 87 & 78 & 83 & 0.89 & 100 & 72 & 90 & 0.94 & 94 & 78 & 88 & 0.92 \\
\hline
\end{tabular}




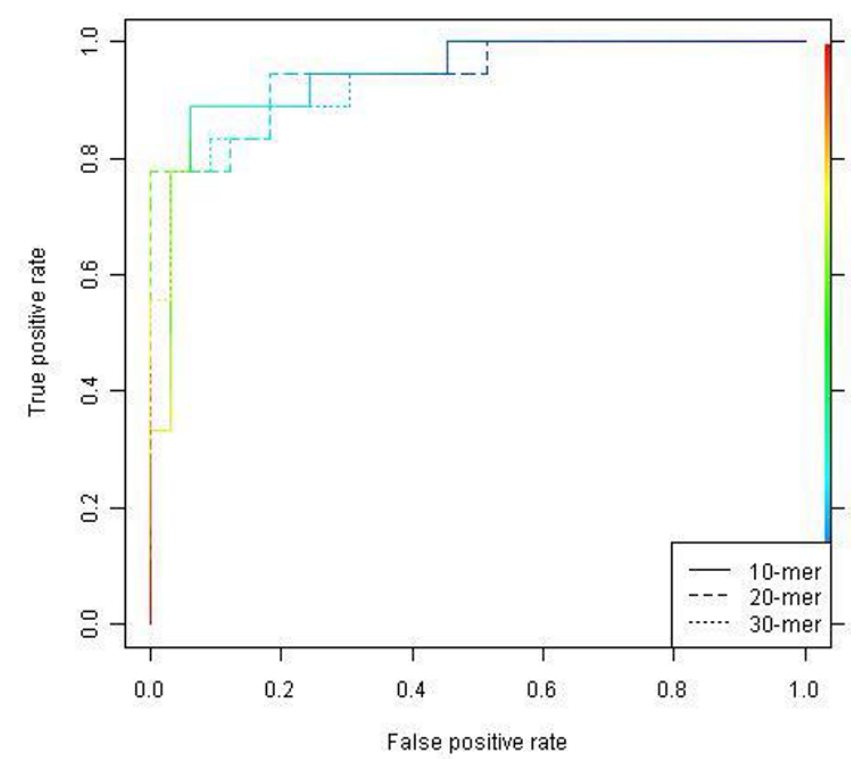

Figure I

RF ROC curves for the 10-mer, 20-mer and 30-mer data sets. The horizontal axes are the false alarm rates (I specificity) and vertical axes are the sensitivity. For specific threshold for discriminating between positive (true sulfotyrosine sites) and negative (unconfirmed sulfotyrosine sites) data points, there will be a pair of these two values, i.e., I - specificity and sensitivity. A pair of values is then represented by a point in this two-dimensional space. Each curve is made by connecting all these points. A model is said to be robust whether its ROC curve is close to the top left corner. The area under a ROC curve is a quantitative indicator of this robustness.

assumes that motif positions are mutually independent with a uniform background distribution which may not be true in most applications [32]. Second, the motifs generated this way are sensitive to experimental errors [33]. Machine learning models, on the other had, are more error-tolerate and have been recognised being capable of generalising well on unseen data.

In the common practice of peptide classification, the input for site prediction is normally a symmetrical peptide of consecutive amino acid resides that flank the potentially modified tyrosine. In this study three peptide sizes have been evaluated and the amino acids have been encoded using a hydrophobicity scale [34]. The encoded numerical data of peptides are then treated as inputs for building prediction models using various machine learning algorithms.

The reason of using a hydrophobicity scale is due to its traditional role in analysing the impact of amino acid hydrophobicity on protein structure and potential sites for protein-protein interactions [35]. Hydrophobic amino acids are generally located in the protein interior whereas hydrophilic amino acids are generally located on the protein surface as targets for binding with other molecules. A protein whose surface is composed of mainly negatively charged amino acids such as glutamate and aspartate will bind to a protein with mainly positively-charged molecules such as lysine and arginine [36-40]. This means that the hydrophobicity scale can be one candidate for encoding amino acids for constructing a predictive model. This study has used the Cornette scale [34].

\section{Results}

There are 18 experimentally verified sulfotyrosine sites and 33 unconfirmed sulfotyrosine sites in 15 blind test sequences. Two inferred sulfotyrosine sites were not used for the evaluation. Table 1 shows the prediction result for these sequences using the Sulfinator. In the table, "Actual" means the experimentally verified sulfotyrosine sites while "Predicted" means the predicted sulfotyrosine sites. "Accession" is the accession number from NCBI database. Numbers in bold face are the sulfotyrosine sites missed from the Sulfinator. Numbers in italic are the false sulfo-

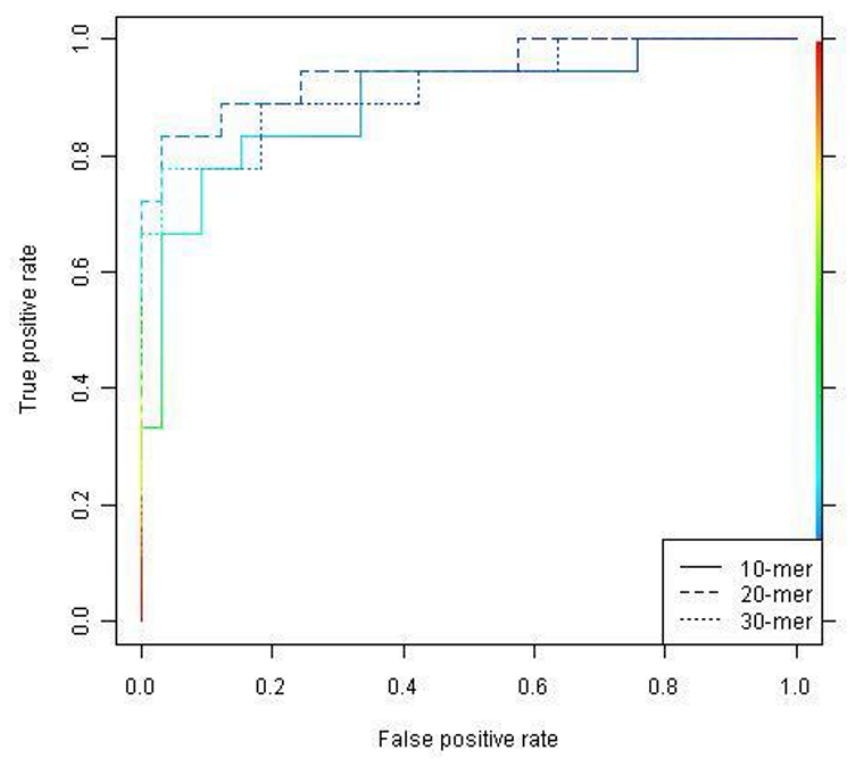

\section{Figure 2}

SVM ROC curves for the 10-mer, 20-mer and 30-mer data sets. The horizontal axes are the false alarm rates (I specificity) and vertical axes are the sensitivity. For specific threshold for discriminating between positive (true sulfotyrosine sites) and negative (unconfirmed sulfotyrosine sites) data points, there will be a pair of these two values, i.e., I - specificity and sensitivity. A pair of values is then represented by a point in this two-dimensional space. Each curve is made by connecting all these points. A model is said to be robust whether its ROC curve is close to the top left corner. The area under a ROC curve is a quantitative indicator of this robustness. 


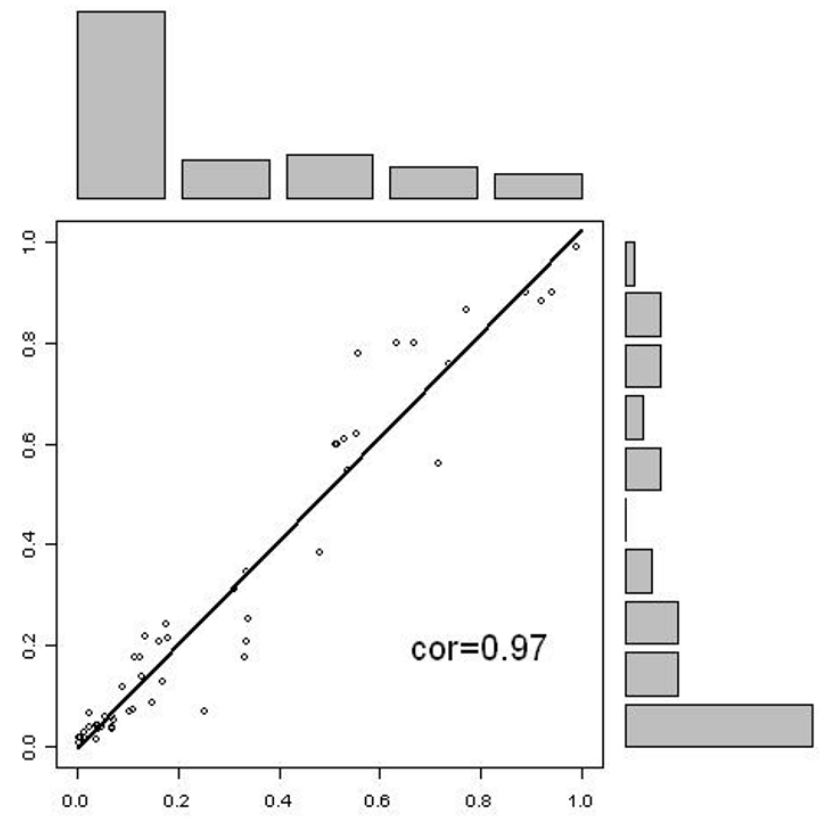

Figure 3

The correlation of the predictions between $10-$ mer model predictions (horizontal axis) and the 20-mer model predictions (vertical axis) for the blind data

set.

tyrosine sites. The total prediction accuracy is $82 \%$ with a specificity of $94 \%$ and a sensitivity of $61 \%$. The sensitivity is $33 \%$ lower than the specificity.

Table 2 shows the prediction performances of all machine learning models constructed in this study. In the Table, the figures in bold face represent the models that outperform the Sulfinator. 10, 20, and 30 represent the peptide sizes. "n.a." represents "not available" because the kNN models have no AUR. The models built using the LDA, QDA, CART, 1NN and ANN5 approaches are not compatible with the Sulfinator. Other approaches generate at least one model as accurate as the Sulfinator depending on peptide sizes. All three RF models outperform the Sulfinator, so do all three $5 \mathrm{NN}$ models. Four models have achieved $>90 \%$ total prediction accuracy with improved specificity and sensitivity. The RF models increase the sensitivity by $22 \%$, the specificity by $3 \%$, and the total prediction accuracy by $10 \%$ compared with the Sulfinator. The 20 -mer SVM model increases the specificity by $6 \%$, the sensitivity by $11 \%$, and the total prediction accuracy by $8 \%$.

The Chi-square test which has been used in bioinformatics [41] is used to evaluate the significance of the improvement of the sensitivity. The test value is $7.93(\mathrm{p}<0.01)$. This represents that the new predictor is able to increase the sensitivity significantly compared with the previous one.

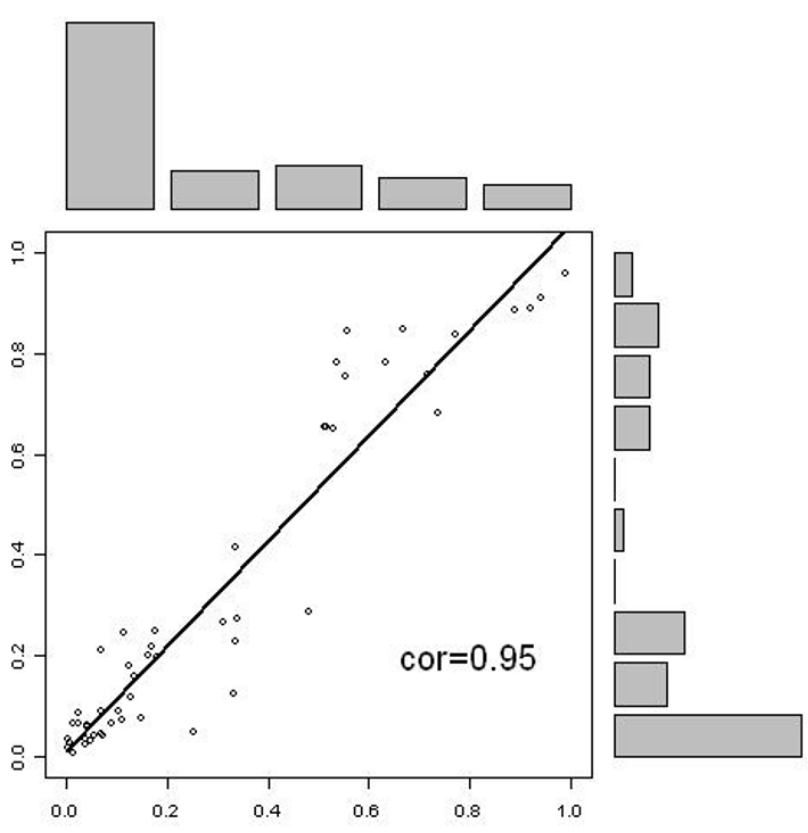

Figure 4

The correlation of the predictions between $10-\mathrm{mer}$ model predictions (horizontal axis) and the 30-mer model predictions (vertical axis) for the blind data set.

Figures 1 and 2 show the ROC curves of the RF and SVM models, respectively. It can be seen that three RF models are consistent while the SVM model built on the 20-mer peptide outperforms the other two SVM models, i.e. the curve is more close to the top-left corner.

\section{Discussion}

In order to investigate the consistency among the RF models, correlation analysis is conducted. Figure 3 shows the correlation analysis of the predictions generated from the 10-mer RF model and 20-mer RF model. The correlation is 0.97 . Figure 4 shows the correlation analysis of the predictions generated from the 10-mer RF model and 30-mer RF model. The correlation is 0.95 . Figure 5 shows the correlation analysis of the predictions generated from the 20mer RF model and 30-mer RF model. The correlation is 0.98 . The high correlation indicates that three RF models are very consistent in prediction demonstrating a high robustness of the algorithm.

Figure 6 shows the ranking results from three RF models (mean decrease Gini gain $[42,43]$ ). It can be seen that residue N1 has been consistently highly ranked. Other residues with higher rank values are C5, C10, and C14. Based on the conditional density functions of $\mathrm{N} 1$ and $\mathrm{C} 1$ shown in Figure 7, it can be seen that residue N1does contribute more to the classification of the two classes of peptides compared with $\mathrm{C} 1$. Because tyrosine sulfation plays a role in protein-protein interaction, several laboratorial works 


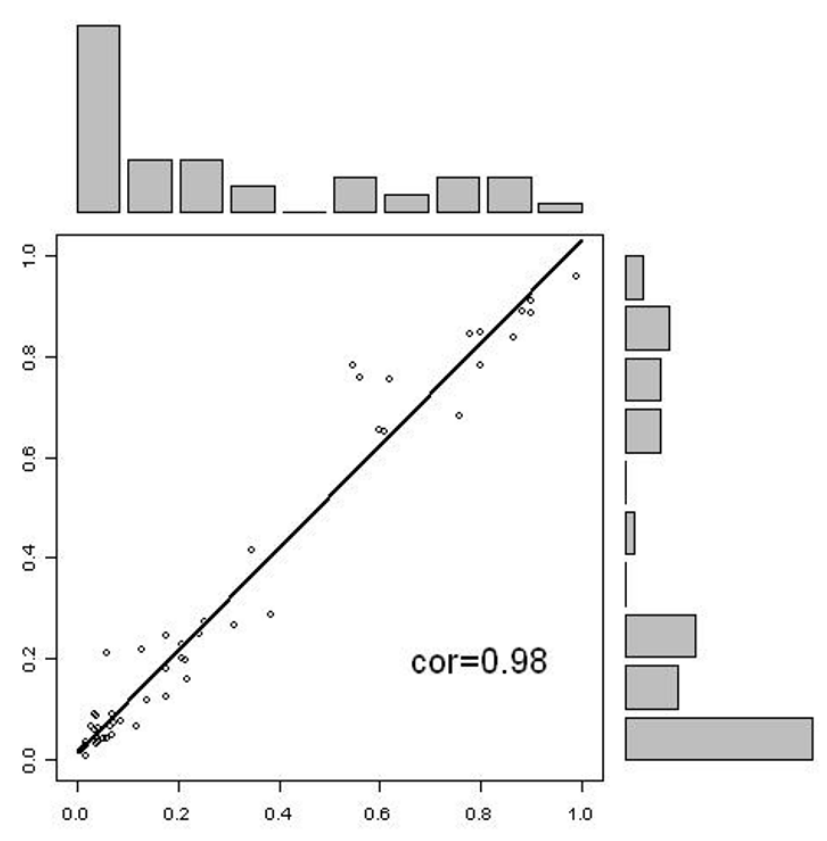

Figure 5

The correlation of the predictions between 20-mer model predictions (horizontal axis) and the 30-mer model predictions (vertical axis) for the blind data set.

have found that $\mathrm{N}$-terminal residues contribute to sulfotyrosines, i.e., the N-terminal domain of sulfotyrosines involve direct protein-protein interaction through a $\mathrm{P}$ selection [44-48].

The major differences between the Sulfinator and the predictors constructed in this paper are the use of different algorithms as well as the different presentation approaches of amino acids to a machine learning model. HMM does not need an encoding process while the predictors constructed in this study use a hydrophobicity scale to encode the amino acids. It is known that the random forest algorithm and the support vector machine algorithm have been well-known in improving the generalisation capability of a model. The significant improvement in the prediction accuracy in blind data these models can result from the use of RF and SVM algorithms and the use of hydrophobicity scale.

Finally, a single RF predictor is built using whole training data coded using 20-mer peptides (excluding any blind sequences). The RF predictor, a $\mathrm{C}$ program which is used to extract 20-mer peptides from a query sequence and encoding peptides using the Cornette scale, the whole training sequences and the blind sequences are available in the web site http://ecsb.ex.ac.uk/sulfotyrosine for free use, where a web tool is also available.
The RF predictor is then used to make prediction on the 15 blind sequences. Its performance is the same as that obtained from cross-validation models. For instance, protein Q9PU41 is a Cholecystokinin with 130 residues. It is related to the release of pancreatic enzymes in the gut [49]. A high degree of identity was found between the sequence from chicken and chinchilla which inferred a homologous function [50]. The sulfotyrosine is the first residue of the peptide Cholecystokinin-7 (112-118). The residue has been accurately predicted by both cross-validation RF predictions and the single RF predictor, as well as using Sulfinator. Protein A2ZBG5 is a Phytosulfokines 2 . Two sulfotyrosine sites have been found in the peptide Phytosulfokine-beta (110-113). This protein is associated with plant cell differentiation, organogenesis, somatic embryogenesis and cell proliferation. The sulfated tyrosine is for binding to a putative membrane receptor [51]. In this case both sites have been accurately predicted using the RF model. However, Sulfinator failed to predict both. The RF predictor failed to predict three sulfotyrosine sites in extracellular proteins (Y21 in P61073 as well as Y97 and Y118 in Q9NZ53) while Sulfinator failed to predict one of these three sites (Y118 in Q9NZ53). Details of the analysis of all the predictions can be seen in Table 3 . In the Table, "Site" represents the experimentally verified sulfotyrosine sites as well as those tyrosine residues which are not experimentally verified sulfotyrosine sites. If the value in the "TURE" column is 1 , it represents that the site is an experimentally verified one. Sulfinator represents the predictions of the Sulfinator tool, where "Y" represents predicted sulfotyrosine sites including false positives. The sites 102 and 131 of Q7 M3V5 are missed experimentally verified sulfotyrosine sites. Blanks represent predicted non- sulfotyrosine sites. "RF1" represents the prediction (posterior probabilities) of cross-validation predictions while "RF2" represents the predictions (posterior probabilities) made by a single RF predictor. "Peptide" represents the segments in which the sulfotyrosine sites sit. "Region" represents the protein in which the sulfotyrosine sites are.

It is also important to see how confident we trust the predictions made by a model and whether this new approach is making a significant contribution to prediction accuracy compared with old models. For this we investigate the properties of the negative and positive predictive powers [52-55]. The negative predictive power measures how likely a negative prediction is true. In other words, it measures the probability that a prediction of unconfirmed sulfotyrosine is a true unconfirmed sulfotyrosine. It is calculated by the fraction of correctly identified unconfirmed sulfotyrosine sites over the total predicted unconfirmed sulfotyrosine sites. The positive predictive power then measures the probability that a sultyrosine prediction is a true sulfotyrosine. This is calculated by the frac- 

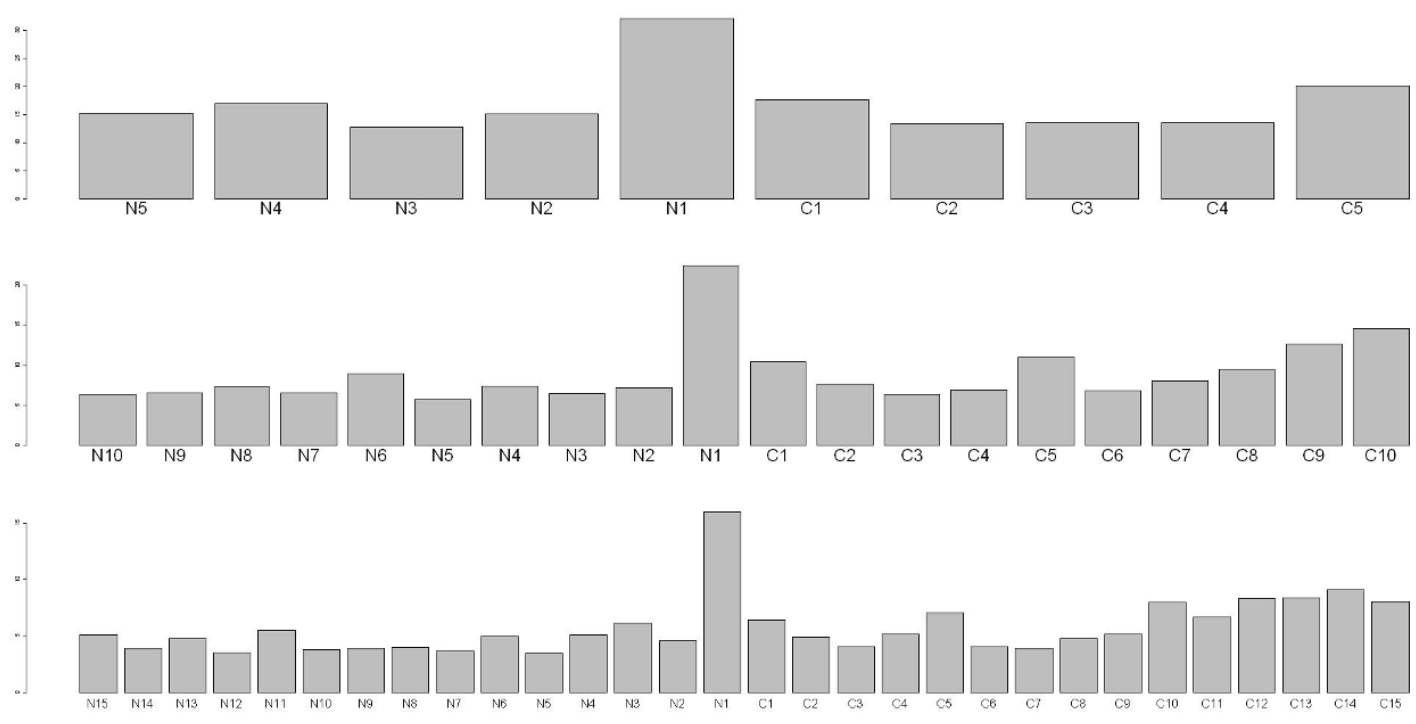

\section{Figure 6}

The ranking results of residues in three RF models. The horizontal axis represents residue positions in peptides. The upper panel is for the 10-mer data, hence having residue positions ranging from $\mathrm{N}_{5}$ to $\mathrm{C}_{5}$. The middle panel is for the 20 -mer data, hence 20 bars. The lower panel is for the 30-mer data, hence 30 bars. The vertical axis indicates the mean decrease Gini measures.

tion of correctly identified true sulfotyrosine sites over the total predicted sulfotyrosine sites. Given the confusion matrix made by testing the blind sequences as in Table 4, we can work out these two measurements. In the Table, "Negative" represents unconfirmed sulfotyrosine and "Positive" represents experimentally verified sulfotyrosine. The left panel is the result obtained when using Sulfi-

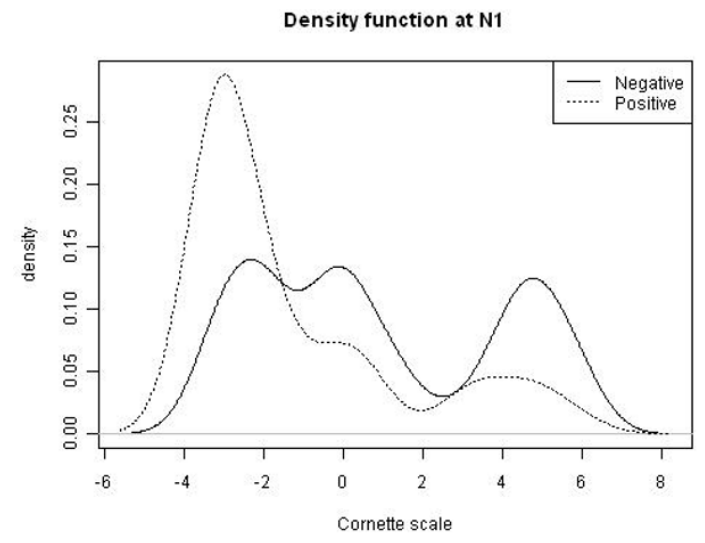

nator while the right panel is the result generated by the $\mathrm{RF}$ model based on the 20-mer data. When using Sulfinator, the negative and the positive predictive powers are $82 \%$ and $85 \%$, respectively. However they are $91 \%$ and $94 \%$, respectively, when using the RF models. It can be seen that the confidence of trusting an unconfirmed sulfotyrosine site has increased by $9 \%$ using the RF models and

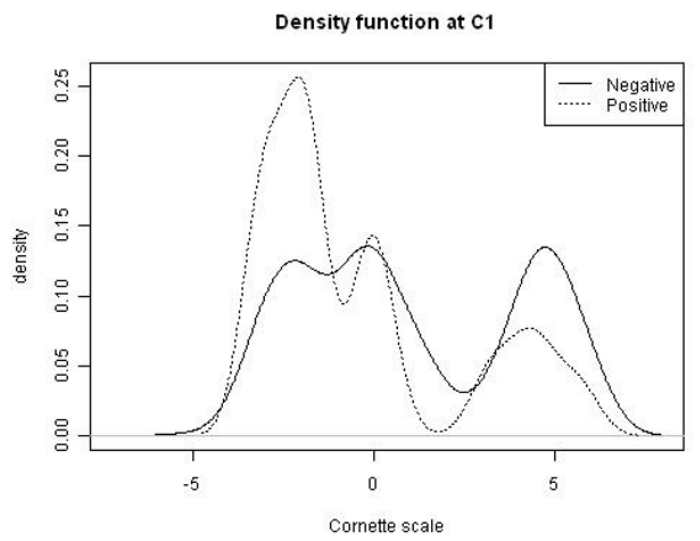

\section{Figure 7}

The conditional density functions drawn at $\mathbf{N}_{1}$ and $\mathbf{C}_{1}$ residues, respectively. The horizontal axes represent the Cornette scale values while the vertical axes represent the density values. The density functions are estimated using the kernel approach using the $R$ stats package with default parameter setting. The graph shows that the density functions drawn at $\mathrm{N}_{1}$ demonstrate a larger separation between two classes while this difference is getting smaller for the residue $C_{l}$, which does not have a high rank value from RF models. Note that negative means unconfirmed sulfotyrosine whilst positive means experimentally verified sulfotyrosine. 
Table 3: The prediction details of I 5 blind testing proteins.

\begin{tabular}{|c|c|c|c|c|c|c|c|}
\hline Protein & Site & TRUE & Sulfinator & RF I & RF2 & Peptide & Region \\
\hline Q9PU4I & 112 & I & $Y$ & 0.9884 & 0.988 & Cholecystokinin-7 & \\
\hline Q9PU4I & 2 & 0 & & 0.1384 & 0.122 & & \\
\hline Q9PU4I & 79 & 0 & & 0.0588 & 0.036 & & \\
\hline P6I073 & 21 & 1 & $\mathrm{Y}$ & 0.2528 & 0.158 & & Extracellular \\
\hline P61073 & 7 & 0 & & 0.176 & 0.092 & & \\
\hline P61073 & 12 & 0 & & 0.128 & 0.264 & & \\
\hline P61073 & 45 & 0 & & 0.0188 & 0.006 & & \\
\hline P61073 & 65 & 0 & & 0.086 & 0.074 & & \\
\hline P61073 & 76 & 0 & & 0.1776 & 0.164 & & \\
\hline P61073 & 103 & 0 & & 0.0084 & 0.008 & & \\
\hline P61073 & 116 & 0 & & 0.04 & 0.036 & & \\
\hline P61073 & 121 & 0 & & 0.034 & 0.03 & & \\
\hline P61073 & 135 & 0 & & 0.0284 & 0.022 & & \\
\hline P61073 & 157 & 0 & & 0.0404 & 0.034 & & \\
\hline P61073 & 184 & 0 & & 0.0408 & 0.026 & & \\
\hline P61073 & 190 & 0 & & 0.0688 & 0.062 & & \\
\hline P61073 & 219 & 0 & & 0.016 & 0.02 & & \\
\hline P61073 & 255 & 0 & & 0.0152 & 0.02 & & \\
\hline P61073 & 256 & 0 & & 0.0388 & 0.04 & & \\
\hline P61073 & 302 & 0 & & 0.0176 & 0.02 & & \\
\hline Q9NZ53 & 97 & I & $Y$ & 0.0692 & 0.076 & & Extracellular \\
\hline Q9NZ53 & 118 & I & & 0.2072 & 0.236 & & \\
\hline Q9NZ53 & 391 & 0 & & 0.0672 & 0.06 & & \\
\hline Q9NZ53 & 481 & 0 & & 0.1168 & 0.112 & & \\
\hline Q9NZ53 & 498 & 0 & & 0.1776 & 0.152 & & \\
\hline Q9NZ53 & 522 & 0 & & 0.0384 & 0.052 & & \\
\hline A2ZBG5 & 110 & 1 & & 0.618 & 0.606 & Phytosulfokine-beta & \\
\hline A2ZBG5 & 112 & I & & 0.7988 & 0.814 & & \\
\hline A2YFB4 & 80 & I & $Y$ & 0.5476 & 0.596 & Phytosulfokine-beta & \\
\hline A2YFB4 & 82 & 1 & & 0.7788 & 0.796 & & \\
\hline Q7 M3V5 & 114 & 1 & $\mathrm{Y}$ & 0.2064 & 0.226 & Callisulfakinin-I & \\
\hline Q7 M3V5 & 2 & 0 & & 0.3116 & 0.358 & & \\
\hline Q7 M3V5 & 12 & 0 & & 0.0608 & 0.064 & & \\
\hline Q7 M3V5 & 56 & 0 & & 0.0528 & 0.044 & & \\
\hline Q7 M3V5 & 64 & 0 & & 0.2184 & 0.232 & & \\
\hline Q7 M3V5 & 65 & 0 & & 0.0348 & 0.038 & & \\
\hline Q7 M3V5 & 81 & 0 & & 0.3856 & 0.378 & & \\
\hline Q7 M3V5 & 102 & 0 & $Y$ & 0.072 & 0.054 & & \\
\hline Q7 M3V5 & $13 \mid$ & 0 & $Y$ & 0.5604 & 0.592 & & \\
\hline P84900 & 62 & I & & 0.608 & 0.582 & Phyllokinin & \\
\hline Q0VTT9 & 62 & 1 & & 0.598 & 0.57 & [Thr6, Vall0]-phyllokinin & \\
\hline POCIV8 & 16 & 1 & $Y$ & 0.8816 & 0.92 & Alpha-conotoxin AnIC & \\
\hline POCIV7 & 16 & 1 & $Y$ & 0.8988 & 0.93 & Alpha-conotoxin AnIA & \\
\hline Q800FI & 62 & 1 & & 0.5984 & 0.57 & [Thr6]-phyllokinin & \\
\hline$P 68116$ & 5 & 1 & Y & 0.7984 & 0.816 & Fibrinopeptide B & \\
\hline P68II6 & 2 & 0 & & 0.3464 & 0.338 & & \\
\hline P68I24 & 4 & 1 & $Y$ & 0.8984 & 0.902 & Fibrinopeptide B & \\
\hline P6812I & 3 & 1 & $\mathrm{Y}$ & 0.7584 & 0.742 & Fibrinopeptide B & \\
\hline P68119 & 6 & 1 & $Y$ & 0.8648 & 0.864 & Fibrinopeptide B & \\
\hline
\end{tabular}

the confidence of trusting a sulfotyrosine site has improved by $9 \%$ as well.

\section{Conclusion}

This paper has presented a new predictor for sulfotyrosine sites in protein sequences. The sequences annotated after 2002 are used as the blind test data for comparing the models constructed using various other machine learning algorithms in this study and Sulfinator, a prediction tool established in 2002. Through evaluation, it has been found that the predictors constructed using the random forest algorithm and the support vector machine algorithm show significantly improved prediction accuracy compared with Sulfinator. The random forest models 
demonstrate consistently better performance. Using the RF models, the sensitivity is increased by $22 \%$, the specificity is increased by $3 \%$, and the total prediction accuracy is increased by $10 \%$ compared with the sulfinator. Both negative and positive predictive powers have been increased by $9 \%$ using the RF models. The 20 -mer RF model is the method of choice for implementing a predictor because it has the highest AUR.

\section{Methods}

\section{Data}

363 proteins with experimentally verified sulfotyrosine sites were collected from NCBI [56]. Two rules were used for pre-processing the data. First, any sequence without an experimentally verified sulfotyrosine was removed. Second, the CD-HIT algorithm [57-59] was used to remove sequences with $\geq 90 \%$ similarity. Applying these two rules gave 94 sequences for the study. Among them, 79 were annotated before 2002 (inclusive) while the rest 15 were annotated after 2002. 79 early annotated sequences were used to train and select a predictor while 15 later annotated sequences were used as blind test data set for the comparison with the Sulfinator. The separation of data in 2002 was because the Sulfinator was developed in 2002. All the sequences annotated after 2002 should therefore be blind to the predictor.

\section{Peptide formation and coding}

All the tyrosines were extracted from the 79 test sequences. Following a common procedure in constructing a PTM site predictor, a peptide was formed symmetrically using both $\mathrm{N}$-terminal and C-terminal consecutive residues flanking a tyrosine. It was denoted by $\mathrm{N}_{\mathrm{m}}-\mathrm{N}_{\mathrm{m}-1^{-}}$ $\ldots \mathrm{N}_{1}-\mathrm{C}_{1}-\ldots \mathrm{C}_{\mathrm{m}-1}-\mathrm{C}_{\mathrm{m}}$. Here, $2 \mathrm{~m}$ was the number of flanking residues in a peptide with $\mathrm{N}_{-k}$ as an $\mathrm{N}$-terminal residue and $\mathrm{C}_{-\mathrm{k}}$ as a C-terminal residue of a tyrosine. Note that the tyrosine in the middle was not used because it was identical in all peptides. Three peptide sizes were used, i.e. $2 \mathrm{~m}$ $=10,20$, and 30. A peptide generated from an experimentally verified sulfotyrosine was labelled as positive while a peptide generated from an unconfirmed sulfated tyrosine (has not yet been declared as a sulfated tyrosine) was labelled as negative. 132 positive and 626 negative nonrepeated peptides were found in 79 sequences. Here, "non-repeated" indicated that any repeated peptide was removed. Each peptide was encoded to a numeric vector using the Cornette hydrophobicity scale.

\section{Model construction}

The machine learning algorithms used in this study were linear discriminant analysis (LDA) $[43,60]$, quadratic discriminant analysis (QDA) $[43,60]$, k-nearest neighbour $(\mathrm{kNN})[43,60]$, classification and regression tree (CART) [61], the random forest algorithm (RF) [62], the support vector machine (SVM) [63] and artificial neural network (ANN) [64]. Because RF is a newly developed machine learning algorithm, a brief description of it is placed below. All these algorithms were available in the R programming environment (built by the R project, http:// www.r-project.org/). The hidden neurons of ANN were 5, 10,15 , and 20 . The numbers of nearest neighbours were $1,3,5,7$, and 9. The distance used in kNN was the Euclidean distance. The radial basis kernel function of the SVM was used with the smoothing parameter as 0.2. The cost parameter of the SVM was 100 . The default parameters of LDA, QDA, CART, and RF were used.

\section{Model evaluation}

Models were evaluated by the sensitivity (Sen, the prediction accuracy of true sulfotyrosine sites), the specificity (Spe, the prediction accuracy of unconfirmed sulfotyrosine sites), the total accuracy (Tot), and receiver operating characteristics (ROC) analysis [65]. ROC was used to measure whether a model was robust. The areas under ROC curves (AURs) were used as a quantitative indicator of model robustness. The five-fold cross-validation approach [66] was used for model evaluation. ROC curves were drawn using the ROCR R package [67] and the area under ROC curves was calculated using the caTools $\mathrm{R}$ package http://cran.r-project.org/web/packages/caTools/ index.html.

\section{The random forest algorithm}

The random forest algorithm is a newly developed machine learning algorithm [62]. The basic idea is to construct many trees using random vectors sampled from a data set. For the kth tree, a random vector is generated independently from the random vectors generated for the past k-1 trees. The remaining data are used for prediction. The approach of sampling random vectors is similar to bootstrap, i.e. the replacement sampling approach, which has also been applied to analysing biological data [68]. For each node in a tree, a small fraction of variables is randomly selected. The best split for the node is based on the prediction error. Each tree is fully grown without pruning.

Table 4: Confusion matrices for Sulfinator and the RF models in this study.

\begin{tabular}{|c|c|c|c|c|c|c|}
\hline & & Sulfinator & & & RF model & \\
\hline & Negative & Positive & & Negative & Positive & \\
\hline Negative & 31 & 2 & $94 \%$ & 32 & I & $97 \%$ \\
\hline \multirow{2}{*}{ Positive } & 7 & 11 & $61 \%$ & 3 & 15 & $83 \%$ \\
\hline & $82 \%$ & $85 \%$ & $82 \%$ & $91 \%$ & $94 \%$ & $92 \%$ \\
\hline
\end{tabular}


$\mathrm{RF}$ is able to provide a number of excellent features, for instance, the capability of handling a large number of variables, ranking the variables, and detecting the interaction among the variables. The algorithm has been recently applied to various biological data mining projects, for example, the prediction of the interactions between HIV1 and human proteins using gene expression data [69], the analysis of differential gene expression [70], the diagnosis of ulcerative colitis based on gene expression data [71], the detection of cancers [72], the prediction of childhood leukaemia using gene expression data [73], and the prediction of protein-protein interactions [74]. All these applications show that the random forest algorithm outperforms some other algorithms.

\section{Authors' contributions}

ZRY collected the data, wrote and debugged $\mathrm{C}$ and $\mathrm{R}$ scripts for model construction and evaluation as well as the presentation of the work, implemented the web tool using JavaScript and Perl, and completed the manuscript preparation.

\section{Acknowledgements}

ZRY thanks Mathew Turnbull and Robin Batten for providing technique support during the implementation of the web tool and Andrew Dalby for critical discussions of the work. The UK high education funded this work.

\section{References}

I. Hille A, Rosa P, Huttner WB: Tyrosine sulfation: a post-translational modification of proteins destined for secretion? FEBS Lett 1984, 177:129-134.

2. Andersen BN: Species variation in the tyrosine sulfation of mammalian gastrins. Gen Comp Endocrinol 1985, 58:44-50.

3. Danielsen EM: Tyrosine sulfation, a post-translational modification of microvillar enzymes in the small intestinal enterocyte. EMBO J 1987, 6:289|-2896.

4. Negishi M, Pedersen LG, Petrotchenko E, Shevtsov S, Gorokhov A, Kakuta Y, Pedersen LC: Structure and function of sulfotransferases. Arch Biochem Biophys 2001, 390:149-I57.

5. Leitinger B, Brown JL, Spies M: Tagging secretory and membrane proteins witha tyrosine sulfation site. The Journal of Biological Chemistry 1984, 269:81 15-8121.

6. Kehoe JW, Bertozzi CR: Tyrosine sulfation: a modulator of extracellular protein-protein interactions. Chem Biol 2000, 7:R57-6I.

7. Seibert C, Sakmar TP: Toward a framework for sulfoproteomics: Synthesis and characterization of sulfotyrosine-containing peptides. Biopolymers 2008, 90:459-477.

8. Liu J, Louie S, Hsu W, Yu KM, Nicholas HB Jr, Rosenquist GL: Tyrosine sulfation is prevalent in human chemokine receptors important in lung disease. Am J Respir Cell Mol Biol 2008, 38:738-743.

9. Plaas $\mathrm{AH}$, West LA, Wong-Palms S, Nelson FR: Glycosaminoglycan sulfation in human osteoarthritis. Disease-related alterations at the non-reducing termini of chondroitin and dermatan sulfate. J Biol Chem 1998, 273: I 2642-I 2649.

10. Coughtrie MW, Bamforth KJ, Sharp S, Jones AL, Borthwick EB, Barker $E V$, Roberts RC, Hume R, Burchell A: Sulfation of endogenous compounds and xenobiotics--interactions and function in health and disease. Chem Biol Interact 1994, 92:247-256.

II. Koo CY, Sen YP, Bay BH, Yip GW: Targeting heparan sulfate proteoglycans in breast cancer treatment. Recent Pat Anticancer Drug Discov 2008, 3: I5I-I58.

12. Dumas NA, He D, Frost AR, Falany CN: Sulfotransferase 2B I b in human breast: differences in subcellular localization in African American and Caucasian women. J Steroid Biochem Mol Biol 2008, I I I: |7|-|77.
13. Hui Y, Yasuda S, Liu MY, Wu YY, Liu MC: On the sulfation and methylation of catecholestrogens in human mammary epithelial cells and breast cancer cells. Biol Pharm Bull 2008, 3 I:769-773.

14. Wang Y, Spitz MR, Tsou AM, Zhang K, Makan N, Wu X: Sulfotransferase (SULT) IAI polymorphism as a predisposition factor for lung cancer: a case-control analysis. Lung Cancer 2002, 35: $137-142$.

15. Sakko AJ, Butler MS, Byers S, Reinboth BJ, Stahl J, Kench JG, Horvath LG, Sutherland RL, Stricker PD, Henshall SM, Marshall VR, Tilley WD, Horsfall DJ, Ricciardelli C: Immunohistochemical level of unsulfated chondroitin disaccharides in the cancer stroma is an independent predictor of prostate cancer relapse. Cancer Epidemiol Biomarkers Prev 2008, I 7:2488-97.

16. Teng YH, Tan PH, Chia SJ, Zam NA, Lau WK, Cheng CW, Bay BH, Yip GW: Increased expression of non-sulfated chondroitin correlates with adverse clinicopathological parameters in prostate cancer. Mod Pathol 2008, 2 I:893-90I.

17. Skandalis SS, Kletsas D, Kyriakopoulou D, Stavropoulos M, Theocharis DA: The greatly increased amounts of accumulated versican and decorin with specific post-translational modifications may be closely associated with the malignant phenotype of pancreatic cancer. Biochim Biophys Acta 2008, 1760: $1217-1225$

18. Li J, Kleeff J, Abiatari I, Kayed H, Giese NA, Felix K, Giese T, Büchler $M W$, Friess $\mathrm{H}$ : Enhanced levels of Hsulf-I interfere with heparin-binding growth factor signaling in pancreatic cancer. Mol Cancer Res 2005, 4:14.

19. Bloushtain N, Qimron U, Bar-Ilan A, Hershkovitz O, Gazit R, Fima E, Korc M, Vlodavsky I, Bovin NV, Porgador A: Membrane-associated heparan sulfate proteoglycans are involved in the recognition of cellular targets by NKp30 and NKp46. J Immunol 2004, I 73:2392-240I.

20. Otvos LJ, Cappelletto B, Varga I, Wade JD, Xiang ZQ, Kaiser K, Stephens LJ, Ertl HC: The effects of post-translational sidechain modifications on the stimulatory activity, serum stability and conformation of synthetic peptides carrying $\mathrm{T}$ helper cell epitopes. Biochim Biophys Acta 1996, I 3 I 3: I I-I9.

21. Wolfender J, Chu F, Ball H, Wolfender F, Fainzilber M, Baldwin M, Burlingame $A$ : Identification of tyrosine sulfation in conus pennaceus conotoxins a-PnIA and a-PnIB: further Investigation of aabile sulfo- and phosphopeptides by electrospray, matrix-assisted laser desorption/lonization (MALDI) and atmospheric pressure MALDI mass spectrometry. J Mass Spectrom 1999, 34:447-454.

22. Coughtrie MW: Sulfation through the looking glass--recent advances in sulfotransferase research for the curious. Pharmacogenomics J 2002, 2:297-308.

23. Buxbaum JD, Geoghagen NS, Friedhoff LT: Cholesterol depletion with physiological concentrations of a statin decreases the formation of the Alzheimer amyloid Abeta peptide. J Alzheimers Dis 200I, 3:221-229.

24. Hasegawa M, Crowther RA, Jakes R, Goedert M: Alzheimer-like changes in microtubule-associated protein Tau induced by sulfated glycosaminoglycans. Inhibition of microtubule binding, stimulation of phosphorylation, and filament assembly depend on the degree of sulfation. J Biol Chem 1997, 272:33| I8-33124.

25. Caporaso GL, Gandy SE, Buxbaum JD, Greengard P: Chloroquine inhibits intracellular degradation but not secretion of Alzheimer beta/A4 amyloid precursor protein. Proc Natl Acad Sci USA 1992, 89:2252-2256.

26. Monigatti F, Gasteiger E, Bairoch A, Jung E: The Sulfinator: predicting tyrosine sulfation sites in protein sequences. Bioinformatics 2002, I 8:769-770.

27. Lin HC, Tsai K, Chang BL, Liu J, Young M, Hsu W, Louie S, Nicholas HB Jr, Rosenquist GL: Prediction of tyrosine sulfation sites in animal viruses. Biochem Biophys Res Comm 2003, 3 I 2: I I 54- I I 58.

28. Poorman RA, Tomasselli AG, Heinrikson RL, Kezdy F]: A cumulative specificity model for protease from human immunodeficiency virus types $I$ and 2 , inferred from statistical analysis of an extended substrate data base. J Biol Chem 1991, 22:|4554-|456|.

29. Yang ZR: Peptide bioinformatics: peptide classification using peptide machines. Methods Mol Biol 2008, 458: I59-I83.

30. Monigatti F, Hekking B, Steen H: Protein sulfation analysis - a primer. Biochimica Biophysica Acta 2006, I 764: I 904-1913. 
31. Schneider TD, Stephens RM: Sequence logos: a new way to display consensus sequences. Nucleic Acids Res 1990, 18:6097-6100.

32. Vacic V, lakoucheva LM, Radivojac P: Two Sample Logo: a graphical representation of the differences between two sets of sequence alignments. Bioinformatics 2006, 22:1536-1537.

33. Freyhult $\mathrm{E}$, Moulton $\mathrm{V}$, Ardell $\mathrm{DH}$ : Visualizing bacterial tRNA identity determinants and antideterminants using function logos and inverse function logos. NAR 2006, 34:905-916.

34. Cornette JL, Cease KB, Margalit H, Spouge JL, Berzofsky JA, DeLisi C Hydrophobicity scales and computational techniques for detecting amphipathic structures in proteins. J Mol Biol 1987 , 1 95:687-693.

35. Creighton TE: Proteins: structures and molecular properties. San Francisco: W. H. Freeman; 1993.

36. Urry DW: The change in Gibbs free energy for hydrophobic association - Derivation and evaluation by means of inverse temperature transitions. Chem Phy Lett 2004, 399:I77-I8I.

37. Georges E: The P-glycoprotein (ABCBI) linker domain encodes high-affinity binding sequences to alpha- and betatubulins. Biochemistry 2007, 46:7337-7342.

38. Neuwirth M, Flicker K, Strohmeier M, Tews I, Macheroux P: Thermodynamic characterization of the protein-protein interaction in the heteromeric Bacillus subtilis pyridoxalphosphate synthase. Biochemistry 2007, 46:5I3I-5I39.

39. Nomura T, Sokabe M, Yoshimura K: Lipid-Protein Interaction of the MscS Mechanosensitive Channel Examined by Scanning Mutagenesis. Biophys J 2006, 91 :2874-288I.

40. Sohn J, Rudolph J: Temperature dependence of binding and catalysis for the Cdc25B phosphatase. Biophys Chem 2006, 1 25:549-555.

41. Pevsner J: Bioinformatics and Functional Genomics. 2nd edition. NY: Wiley-Blackwell; 2003.

42. Archer KJ, Kimes RV: Empirical characterization of random forest variable importance measures. Computational Stat \& Dato analysis 2007, 52:2249-2260.

43. Ripley BD: Pattern Recognition and Neural Networks. Cambridge: Cambridge University Press; 1996.

44. Wilkins PP, Moore KL, McEver RP, Cummings RD: Tyrosine sulfation of P-selectin glycoprotein ligand-I is required for high affinity binding to P-selectin. J Biol Chem 1995, 270:22677-22680.

45. Pouyani T, Seed B: PSGL-I recognition of P-selectin is controlled by a tyrosine sulfation consensus at the PSGL-I amino terminus. Cell 1995, 83:333-343.

46. Sako D, Comess KM, Barone KM, Camphausen RT, Cumming DA, Shaw GD: A sulfated peptide segment at the amino terminus of PSGL-I is critical for P-selectin binding. Cell 1995, 83:323-331.

47. Somers WS, Tang J, Shaw GD, Camphausen RT: Insights into the molecular basis of leukocyte tethering and rolling revealed by structures of P- and E-selectin bound to $\operatorname{SLe}(X)$ and PSGL-I. Cell 2000, 103:467-479.

48. Hoffhines AJ, Damoc E, Bridges KG, Leary JA, Moore KL: Detection and purification of tyrosine-sulfated proteins using a novel anti-sulfotyrosine monoclonal antibody. J Biol Chem 2006, 28I:37877-37887.

49. Furuse $M$ : Release and endogenous actions of the gastrin cholecystokinin (CCK) family in the chicken. J Exp Zool 1999, 283:448-454.

50. Fan ZW, Eng J, Miedel M, Hulmes JD, Pan YC, Yalow RS: Cholecystokinin octapeptides purified from chinchilla and chicken brains. Brain Res Bull 1987, I 8:757-760.

5I. Matsubayashi Y, Takagi L, Sakagami Y: Phytosulfokine-alpha, a sulfated pentapeptide, stimulates the proliferation of rice cells by means of specific high- and low-affinity binding sites. PNAS 1997, 94: I3357-I3362.

52. Barkley RA, Grodzinksi GM: Are tests of frontal lobe functions useful in the diagnosis of Attention Deficit Disorders? The Clinical Neurologist 1994, 8:121-139.

53. Ellwood RW: Clinical discriminations and neuropsychological tests: An appeal to Bayes' theorem. The Clinical Neuropsychologist 1993, 7:224-233.

54. Matier-Sharma K, Perachio N, Newcorn JH, Sharma V, Halperin JM: Differential diagnosis of ADHD: Are objective measures of attention, impulsivity, and activity level helpful? Child Neuropsychology 1995, I: I I8-127.
55. Wherry JN, Paal N, Jolly JB, Balkozar A, Holloway C, Everett B, Vaught $L$ : Concurrent and discriminant validity of the Gordon Diagnostic System: A preliminary study. Psychology in the Schools 1993, I:29-36.

56. Ostell JM, Kans JA: The NCBI data model. Methods Biochem Anal 1998, 39:121-144.

57. Li W, Jaroszewski L, Godzik A: Clustering of highly homologous sequences to reduce the size of large protein databases. Bioinformatics 200I, 17:282-293.

58. Li W, Jaroszewski L, Godzik A: Tolerating some redundancy significantly speeds up clustering of large protein databases. Bioinformatics 2002, 18:77-82.

59. Li W, Godzik A: Cd-hit: a fast program for clustering and comparing large sets of protein or nucleotide sequences. Bioinformatics 2006, 22: 1658-1660.

60. Venables WN, Ripley BD: Modern Applied Statistics with S. 4th edition. NewYork: Springer; 2002.

6I. Breiman L, Friedman JH, Olshen RA, Stone C): Classification and Regression Trees. Wadsworth 1984.

62. Breiman L: Random forests. Machine Learning 200I, 45:5-32.

63. Vapnik V: The Nature of Statistical Learning Theory. New York: Springer-Verlag; 1995.

64. Rumelhart DE, McClelland JL: Parallel Distributed Processing: Foundations. Volume I. Cambridge, MA, USA: The MIT Press; 1987.

65. Metz CE: Basic principles of ROC analysis. Seminars in Nuclear Medicine 1978, 8:283-288.

66. Efron B: Nonparametric estimates of standard error: The jackknife, the bootstrap and other methods. Biometrika 198I, 68:589-599.

67. Sing $T$, Sander $O$, Beerenwinkel $N$, Lengauer $T$ : ROCR: visualizing classifier performance in R. Bioinformatics 2005, 21:3940-394I.

68. Efron $B$, Halloran $\ddagger$, Holmes $S$ : Bootstrap confidence levels for phylogenetic trees. PNAS 1996, 93: I3429-13434.

69. Tastan O, Oi Y, Carbonell JG, Klein-Seetharaman J: Prediction of interactions between HIV-I and human proteins by information integration. Pac Symp Biocomput 2009:5 16-527.

70. $\mathrm{Wu} X Y, \mathrm{Wu} Z \mathrm{ZY}$, Li K: Identification of differential gene expression for microarray data using recursive random forest. Chin Med J 2008, I 2 I:2492-2496.

71. Olsen J, Gerds TA, Seidelin JB, Csillag C, Bjerrum JT, Troelsen JT, Nielsen $\mathrm{OH}$ : Diagnosis of ulcerative colitis before onset of inflammation by multivariate modeling of genome-wide gene expression data. Inflamm Bowel Dis 2009, 15:1032-1038.

72. Abba MC, Sun H, Hawkins KA, Drake JA, Hu Y, Nunez MI, Gaddis S, Shi T, Horvath S, Sahin A, Aldaz CM: Breast cancer molecular sig. natures as determined by SAGE: correlation with lymph node status. Mol Cancer Res 2007, 5:88I-890.

73. Hoffmann K, Firth MJ, Beesley AH, de Klerk NH, Kees UR: Translating microarray data for diagnostic testing in childhood leukaemia. BMC Cancer 2006, 6:229.

74. Qi Y, Bar-Joseph Z, Klein-Seetharaman J: Evaluation of different biological data and computational classification methods for use in protein interaction prediction. Proteins 2006, 63:490-500.

Publish with Bio Med Central and every scientist can read your work free of charge

"BioMed Central will be the most significant development for disseminating the results of biomedical research in our lifetime. "

Sir Paul Nurse, Cancer Research UK

Your research papers will be:

- available free of charge to the entire biomedical community

- peer reviewed and published immediately upon acceptance

- cited in PubMed and archived on PubMed Central

- yours - you keep the copyright 